\title{
L'HOMME L'Homme
}

Revue française d'anthropologie

160 | octobre-décembre 2001

Droit, coutume, mémoire

\section{Gary M. Feinman \& Joyce Marcus, eds, Archaic States}

(Published with the support from The Brown Fondation of Houston, Texas) Santa Fe, School of American Research Press, 1998, XIV + 427 p., réf., index, ill., tabl. (« School of American Research Advanced Seminar Series »)

\section{Claude-François Baudez}

\section{OpenEdition}

\section{Journals}

Édition électronique

URL : http://journals.openedition.org/lhomme/7989

DOI : 10.4000/lhomme.7989

ISSN : 1953-8103

Éditeur

Éditions de l'EHESS

Édition imprimée

Date de publication : 1 janvier 2001

Pagination : 299-300

ISBN : 2-7132-1391-6

ISSN : 0439-4216

Référence électronique

Claude-François Baudez, « Gary M. Feinman \& Joyce Marcus, eds, Archaic States », L'Homme [En ligne],

160 | octobre-décembre 2001, mis en ligne le 31 mai 2007, consulté le 24 septembre 2020. URL :

http://journals.openedition.org//homme/7989; DOI : https://doi.org/10.4000//homme.7989

Ce document a été généré automatiquement le 24 septembre 2020.

(c) École des hautes études en sciences sociales 


\section{Gary M. Feinman \& Joyce Marcus, eds, Archaic States}

(Published with the support from The Brown Fondation of Houston, Texas) Santa Fe, School of American Research Press, 1998, XIV + 427 p., réf., index, ill., tabl. (« School of American Research Advanced Seminar Series »)

\section{Claude-François Baudez}

1 LES PARTICIPANTS au séminaire «États archaïques » de la School of American Research, qui s'est tenu fin 1992, souhaitaient mettre l'accent non pas tant sur la définition de l'État archaïque que sur les modalités de son fonctionnement, non pas tant sur le concept que sur la diversité et l'instabilité de ses formes à travers le monde, cette approche encourageant la production d'exemples et de comparaisons.

Quels que soient les critères retenus pour définir l'État, on peut se demander comment l'archéologue parvient à les identifier sur le terrain. En comparant les plans de palais, de temples et de tombes royales provenant de divers États archaïques, Kent Flannery dégage des constantes qui renvoient surtout à l'existence d'un pouvoir royal et à une religion officielle. L'étude de la structure de l'habitat permet aussi de savoir si on peut distinguer les quatre niveaux d'établissements requis (village, gros village, ville, " capitale ») pour identifier un État. En revanche, la fouille est souvent impuissante à démontrer l'existence d'une administration ou de l'emploi légal de la force.

3 À l'aide d'exemples pris dans diverses régions du globe, Joyce Marcus montre que les États archaïques connaissent des cycles répétés de consolidation, expansion et dissolution, et aborde la question de la genèse de l'État. Bien souvent, celui-ci naît quand une chefferie réussit à dominer ses voisins. Par contre, un groupe de chefferies ne devient jamais un groupe de "cités-États", concept critiqué par l'auteur. Pendant les périodes de décentralisation, on voit se manifester, de la part des unités politiques qui résultent de l'éclatement d'une hégémonie, de nombreuses tentatives pour créer de plus grandes unités par alliance ou conflit.

4 Gary Feinman s'interroge sur les rapports entre la taille des unités politiques et la forme de leur gouvernement. Y a-t-il un seuil démographique et/ou territorial au-delà 
duquel une chefferie devient un État? On estime que l'information peut atteindre tous les membres d'une communauté si celle-ci comprend moins de 2500 personnes; audelà de ce chiffre, deux niveaux au moins de décision sont nécessaires pour assurer la transmission de la communication. Feinman pense que les États pré-industriels étaient probablement de petite taille mais faisaient partie de systèmes plus larges.

5 Critiquant la théorie des systèmes, qui a tendance à voir dans l'État un simple mécanisme de contrôle du traitement de l'information, Richard Blanton trouve que l'on accorde trop d'importance à la centralisation politique dans la genèse et le fonctionnement des États archaïques. Il insiste sur le rôle de la compétition pour le pouvoir dans les sociétés complexes et distingue sources de pouvoir et types de pouvoir. Les concepts et les définitions qu'il propose devraient, selon lui, faciliter l'analyse comparative de l'économie politique des groupes.

6 Avec Henry Wright et son analyse des États uruk du sud-ouest de l'Iran au IV millénaire, on aborde l'étude de cas. L'auteur compare trois régions (Suse, Deh Luran et Izeh) différentes par leur environnement et leurs productions, la seconde exportant ses produits dans les deux autres. La présentation de leurs histoires respectives est intéressante, mais on ne voit pas bien où l'auteur veut en venir et ses conclusions ne sont guère éclairantes.

7 L'analyse comparative souhaitée par les organisateurs du séminaire est fort bien illustrée par l'article de John Baines et Norman Yoffee, «Order, legitimacy and wealth in ancient Egypt and Mesopotamia ». Dans ces deux civilisations peu éloignées géographiquement, l'État est apparu sensiblement en même temps, vers 3100 av. J.-C. La différence principale qui oppose l'Égypte à la Mésopotamie est que, dans la première, l'unité politique et la civilisation coïncident, alors que la seconde n'est jamais parvenue à l'unification politique avant la conquête perse du premier millénaire avant notre ère. En outre, les deux zones diffèrent quant à la royauté, l'urbanisation et la nature des sources. Dans les deux cas cependant, le thème de l'ordre joue un rôle fondamental en légitimant l'inégalité ; quant à la richesse, elle permet de développer et de préserver des formes sociales complexes. Les auteurs insistent sur l'importance de la « high culture » dans la formation de la civilisation et l'organisation de l'État. Limiter à une petite élite (inner elite) l'accès aux objets d'art, au savoir et à l'écriture est une stratégie qui a été très performante dans ces deux États.

8 Pour Gregory Possehl, la civilisation de l'Indus, aussi complexe qu'elle ait été sur le plan socioculturel, ne peut être considérée comme un État, essentiellement en raison de l'absence d'architecture monumentale religieuse (temples) ou civile (palais). Et ce malgré l'existence de trois centres urbains importants (Mohenjo-Daro, Harappa et Ganweriwala), une écriture, un commerce à longue distance, des classes sociales, et un style artistique répandu sur un million de kilomètres carrés pendant six siècles.

Craig Morris expose les diverses stratégies de l'État inka visant à absorber de nombreux groupes locaux sans user de la violence: participation aux cérémonies, architecture publique à l'image d'une structure politique dominante, centre administratif pour ordonner hiérarchiquement diverses communautés, redistribution économique, contrôle et ritualisation de conflits (batailles rituelles).

10 L'article de David Webster, hors sujet, compare la guerre chez les Maya et les Polynésiens. Les sociétés comme les sources sont sensiblement différentes et c'est sans doute ce qui explique l'échec de l'entreprise. On ne doit comparer que ce qui est 
comparable, et les exemples polynésiens n'éclairent en rien la pratique de la guerre chez les Maya.

11 Bien que les articles qui le constituent soient de valeur et d'intérêt inégaux, cet ouvrage a pour mérite essentiel de montrer la relativité de concepts tels que ceux d'« État » et de "chefferie». Les discussions sur le passage de la chefferie à l'État ou sur le statut d'État à donner à telle ou telle société sont loin d'être closes. On constate qu'il est rare qu'un État possède les mêmes critères que ceux retenus pour d'autres. Ainsi, Teotihuacan est plus urbaine qu'une cité maya, mais l'absence manifeste d'un roi y étonne. Quant aux Maya eux-mêmes, ils se rapprochent de l'Égypte par leurs rois, mais de la Mésopotamie par la prolifération d'unités politiques concurrentes.

\section{AUTEUR}

\section{CLAUDE-FRANÇOIS BAUDEZ}

CNRS, Université Paris-X, Maison de l'archéologie et de l'ethnologie, Nanterre. 